Instituto Internacional de Investigación y Desarrollo Tecnológico Educativo INDTEC, C.A.

DOI: https://doi.org/10.29394/Scientific.issn.2542-2987.2021.6.19.19.366-382

OAI-PMH: http://www.indteca.com/ojs/index.php/Revista Scientific/oai

Ensayo Original / Original Essay

\title{
La Bioética: Un Elemento Fundamental en la Práctica de Enfermería
}

Autor: Rubén Eliut Hernández Ortega

Universidad de las Ciencias de la Salud, UCS

rubenho1977@gmail.com

Caracas, Venezuela

https://orcid.org/0000-0003-3844-278X

\section{Resumen}

El presente ensayo tiene como propósito dar a conocer uno de los elementos indispensables de la Práctica de Enfermería, como lo es la Bioética, ya que los aspectos éticos de la vida, el deber ser y el por qué hacer de los actos humanos, la aplicación que rige la conducta y el comportamiento social, conllevan a revisiones continuas de los problemas éticos colectivos y medioambientales que afectan a las personas en sus diversos contextos. Por lo tanto, el cuidado humano, es un proceso reflexivo, intersubjetivo, de sensaciones y experiencias compartidas, entre el profesional de enfermería y las personas en procesos de salud o enfermedad, no debe ser contrario a los aspectos éticos de la vida y los derechos humanos de las personas, familias y comunidades, aplicando los valores y transversalizando la acción, desde la aceptación y el respeto por los modos de vida, sus costumbres, ideologías, orientaciones sexuales, creencias y religiones, de modo que se comprenda al medio ambiente donde habitan y se desenvuelven como parte fundamental de sus existencias. Se toman como referentes a Schmidt (2008); Jahr (2013); Wilches (2011); y Henderson (1961).

Palabras clave: bioética; personal paramédico; servicio de enfermería.

Cómo citar este ensayo:

Hernández, R. (2021). La Bioética: Un Elemento Fundamental en la Práctica de Enfermería. Revista Scientific, 6(19), 366-382, e-ISSN: 2542-2987. Recuperado de: https://doi.org/10.29394/Scientific.issn.2542-2987.2021.6.19.19.366-382

Fecha de Recepción: 31-07-2020
Fecha de Aceptación:

05-12-2020
Fecha de Publicación: 05-02-2021 


\title{
Bioethics: A Fundamental Element in Nursing Practice
}

\begin{abstract}
The purpose of this essay is to present one of the essential elements of Nursing Practice, such as Bioethics, since the ethical aspects of life, the duty to be and the why of human acts, the application that governs conduct and social behavior, lead to continuous reviews of collective ethical and environmental problems that affect people in their various contexts. Therefore, human care is a reflective, intersubjective process, of shared sensations and experiences, between the nursing professional and people in health or disease processes, it should not be contrary to the ethical aspects of life and rights human rights of people, families and communities, applying the values and mainstreaming the action, from the acceptance and respect for the ways of life, their customs, ideologies, sexual orientations, beliefs and religions, so that the environment is understood where they inhabit and develop as a fundamental part of their existence. They are taken as referring to Schmidt (2008); Jahr (2013); Wilches (2011); and Henderson (1961).
\end{abstract}

Keywords: bioethics; paramedical personnel; nursing service.

How to cite this essay:

Hernández, R. (2021). Bioethics: A Fundamental Element in Nursing Practice. Revista Scientific, 6(19), 366-382, e-ISSN: 2542-2987. Recovered from: https://doi.org/10.29394/Scientific.issn.2542$\underline{2987.2021 .6 .19 .19 .366-382}$

Date Received: 31-07-2020
Date Acceptance:

05-12-2020
Date Publication: 05-02-2021 


\section{Introducción}

En este ensayo, se pretende dar a conocer uno de los elementos indispensables en la realización de la Práctica de Enfermería, como lo es la Bioética, ya que ésta es considerada como un principio filosófico subyacente en la actuación de los profesionales de la enfermería, enmarcándose en el deber ser y el por qué hacer, debido a que es una disciplina que estudia los aspectos éticos de las ciencias de la vida, los actos humanos, la aplicación que rige la conducta y el comportamiento de y para la vida, conllevando además a la revisión continua de los problemas éticos colectivos del mundo humano, así como los problemas sociales y medioambientales que afectan a las personas, y sus modos de vida, impactando de manera directa en la salud integral: relaciones humanas y las convivencias, el entorno, las condiciones culturales e ideológicas y su desenvolvimiento sin limitaciones, dentro de la sociedad donde vive.

Bajo estos fundamentos, se sustenta la práctica de enfermería, ya que el cuidado está estrechamente relacionado con los principios que rigen la conducta humana y el respeto a la vida, como un derecho humano irrenunciable, a partir del compromiso ético hacia las personas que atiende, incluyendo a los familiares de éstas, y al contexto social que conforma su hábitat.

Por ende, el principal factor para llevar a cabo el cuidado humano, es reconocer los derechos universales de las personas, sus apegos culturales, espirituales, étnicos, religiosos e ideológicos, que deben ser respetados por los enfermeros y las enfermeras. Destacando que el razonamiento bioético, inicia con la aplicación de valores éticos y morales, como un método que ayuda a reflexionar sobre las acciones conjuntas de la vida cotidiana, concatenadas a proporcionar respuestas humanas que satisfagan las necesidades $\mathrm{e}$ interrelacionen todos los elementos del ambiente, para favorecer la vida y la salud colectiva. 
Es por lo que, los aspectos relacionados con la aplicación de normas éticas y bioéticas responden a un principio humano que hace manifiesto la concientización de los actos humanos, la puesta en práctica de valores y su incidencia en la salud colectiva e individual de las personas, como un soporte de reciprocidad y a la vez de protección ambiental, que incide en el bienestar humano.

El quehacer enfermero se centra en el respeto por la vida, las personas, sus costumbres, sus realidades y cualquier otro aspecto humano que refleje la reflexión individual de su actuación, ya que la asistencia terapéutica es realizada a las personas, bajo un trato de respeto a su dignidad, sus vidas, y todas sus condiciones humanas, por ello la bioética forma parte esencial del humanismo.

Es importante resaltar, que la aplicación de los principios éticos de la vida, en la práctica de enfermería, abarca una serie de elementos concatenados entre sí, como es la disposición que se tiene para realizar los cuidados de forma integral, haciendo uso de la vocación de servicio, los valores como fuente de importancia y reconocimiento hacia las personas, la consciencia, que de acuerdo con el Diccionario de la Real Academia Española (RAE, 2020): es el "conocimiento inmediato o espontáneo que el sujeto tiene de sí mismo, de sus actos y reflexiones" (pág. 1); con el fin de reflexionar continuamente sobre lo que se hace y, juzgar de forma positiva la actuación profesional, en pro de mejorarla progresivamente.

De igual forma, es importante señalar que los fundamentos jurídicos del ejercicio de la enfermería, describen una actuación donde se destaca que la razón de ser está centrada en el cuidado a las personas, por ello, los enfoques ontológico y epistémico la catalogan como una profesión humanista, que cuida y preserva la vida y la salud, demuestra respeto por la dignidad de las personas en todas las etapas del desarrollo, tomando en cuenta la promoción de la salud y la calidad de vida, además de su participación en la prevención de 
enfermedades y en la recuperación, rehabilitación y aplicación de tratamientos, utilizando los principios humanísticos, éticos y morales, en su práctica diaria.

Por esta razón se concibe que, el profesional de enfermería más que aplicar el conocimiento técnico-científico, tiene el deber fundamental de modelar una ética que considere y valore al ser humano en sus dimensiones biológicas, psicológicas, sociales y espirituales, como exigencia de la práctica humanista de enfermería.

Al respecto, Vargas y Cortes (2010): consideran que la bioética es vista como "la conciencia de las ciencias médicas y biológicas, práctica dinámica, racional y reguladora de los valores éticos y deontológicos [...]" (pág. 43). Es decir, que el uso de la bioética en las ciencias médicas, es de vital importancia en la Enfermería, ya que se enmarca en regular, incentivar y ayudar en el reconocimiento de aspectos cognitivos que mejoren la práctica, a través de procesos reflexivos, con el fin de asumir y expresar de forma actitudinal, los valores éticos y morales que definen esta profesión.

Asimismo, la Federación de Colegios de Profesionales de la Enfermería (FCPERBV, 2008a): en el Código Deontológico de los Profesionales de la Enfermería, artículo nro. 2, hace énfasis en la encomienda social que rige la actuación de enfermería, como principios y competencias de la acción humana realizada. El mismo fundamento jurídico, para la (FCPERBV, 2008b): hace énfasis en que "las normas éticas y/o morales, contenidos en este código son de obligatorio cumplimiento para todas y todos los profesionales de la enfermería en todos los ámbitos de su vida pública y privada" (art. 4);

Por esta razón, se pone de manifiesto que estos profesionales deben realizar su praxis profesional, rigiéndose por el código deontológico del ejercicio de la enfermería, del cual se desprende la bioética como el estudio de la conducta y la vida humana en todas las etapas del desarrollo y desde las dimensiones humanas anteriormente descritas, ya que son normas establecidas para la atención asistencial y el cuidado de enfermería. 


\section{Desarrollo}

La Bioética, está considerada como un principio fundamental en la práctica de enfermería, ya que su fin de aplicación, consiste en velar por los aspectos humanos que rigen la conducta y la vida de las personas, haciendo visible la concientización de los actos humanos, por medio de la aplicación de valores que ayuden a proteger la salud colectiva e individual de éstas, como un soporte de protección correlacional con el medio ambiente.

Por lo tanto, es importante destacar que ésta se fundamenta en los principios bioéticos y el valor humano, como elementos indisolubles que dependen de los preceptos humanos, enmarcados en la plena consciencia del respeto por la dignidad de las personas y la aceptación de estas, demostrada a través de una disposición bondadosa y amorosa, durante el quehacer profesional. Bajo estos términos, se considera lo señalado por Terán y Rico (2018): "la enfermería se caracteriza por ser ética y humanista, por el trato directo con su profesión y praxis social, pues el enfermero(a) transmite empatía, respeto, responsabilidad, entre otros valores que discurren a una ética profesional" (pág. 71).

En este sentido, se destaca que la esencia del cuidado humano no debe ser contraria a los aspectos éticos de la vida y los derechos humanos de las personas, familias y comunidades, ya que los valores están inmersos en la práctica, conjugándose como un todo que transversaliza la acción, desde la aceptación y el respeto por los modos de vida, sus costumbres, ideologías, orientaciones sexuales, creencias y religiones, comprendiendo al medio ambiente donde habitan y se desenvuelven como parte fundamental de sus existencias, debido a que tienen espiritualidad y convicciones personales únicas, cuya esencia parte del humanismo, como un aspecto intrínseco de las relaciones humanas.

Con relación al tema, Jahr (2013): considera a la bioética como "la necesidad de encontrar un equilibrio entre los valores y los objetivos de vida 
de los seres vivientes en su lucha por sobrevivir y en sus necesidades de alimento, espacio y desarrollo" (pág. 187). Además, el autor menciona la interacción estrecha entre el cuidado de la salud individual y el cuidado de la salud pública, haciendo énfasis en que esto constituye un aspecto ético de responsabilidad que deben tener las personas con el ambiente donde se desenvuelve su vida.

De igual forma, Schmidt (2008a), define desde una perspectiva sistémica y abierta, la capacidad para lograr acciones efectivas en la vida y el trabajo, estableciendo a los:

Principios y Valores. Con los que predicó que la concienzación moral y la ética deben estar presentes en las personas, de forma que les permita determinar criterios y patrones de referencia que faciliten el ejercicio de conductas congruentes con la cultura del trabajo y calidad de vida que las sociedades las promuevan y esperen que ellas mismas contribuyan a preservar al hombre y su entorno (pág. 10).

De hecho, Schmidt (2008b): abordó este aspecto en sus primeros trabajos de investigación, basándose en los procedimientos médicos, de los cuales aludía a una combinación de ciencia y filosofía. Sin embargo, en trabajos posteriores, destaca que la concienciación moral y la ética deben estar inmersas en las personas, por lo que es oportuno resaltar que éste proceso debe lograr construir juicios y estándares de referencia en los enfermeros y las enfermeras, de modo que su actuación se enmarque en un entendimiento pleno del ejercicio profesional, proporcionen conductas que promuevan, desde su quehacer, calidad de vida y bienestar colectivo, a través de la contribución de las personas, familias y comunidades en la preservación de su especie, su salud, sus vidas y su entorno medioambiental, como parte de un todo.

En este sentido, la bioética, no sólo actúa en el significado del peligro que ha representado el avance de la tecnociencia en la conservación de vida, 
el deber moral hacia el futuro, el control de la tecnología y los procesos técnicos, surgiendo la necesidad de un esfuerzo interdisciplinario para responder a los problemas éticos que emanan del ámbito clínico, biomédico y biotecnológico, sino en el sentido humano y su correlación con la subsistencia de esta especie.

Por lo tanto, éstos profesionales tienen como corresponsabilidad social, aplicar los valores bioéticos y, además, velar por su cumplimiento, debido a que el proceso enfermero, centra su acción en cuidar la vida de las personas, desde su concepción física, mental, social, espiritual y cultural, concibiendo a los valores éticos y morales como elementos que fundamentan el cuidado, hecho concatenado con lo expresado por Wilches (2011): "el gran reto que tiene ante sí la bioética contemporánea es desarrollar el camino de reflexión crítica abarcadora, que integre ciencia y vida, conocimiento y moralidad, los problemas vitales de la humanidad con perspectivas de presente y futuro" (pág. 78).

Es por ello que esta ciencia, no sólo debe guiar una preocupación moral en las implicaciones éticas de los avances biomédicos, sino en las problemáticas globales, el crecimiento social, las transformaciones influenciadas por las mismas personas, las cuales ponen en riesgo la conservación de la vida, como es la contaminación generalizada, los altos índices de pobreza, desigualdad socio-económica y todos aquellos aspectos que amenazan y comprometen la salud integral, lo cual deben llevar a la realización de actos humanos que promuevan la dignidad y el respeto por la vida humana y el medioambiente.

Por lo tanto, en esta investigación, la bioética en enfermería es vista desde los aspectos fundamentales del respeto a la vida de las personas, más allá de un dilema ético, una concepción humana, que deriva en una atención terapéutica e interpersonal de ayuda, cuyo fin es hacerlas consciente de los procesos inherentes al desarrollo de sus vidas, en todas las etapas del 


\section{Ensayo Original / Original Essay}

crecimiento, la significación de los procesos de salud y de enfermedad, las condiciones y modos de vida elegibles, incluyendo la muerte como un proceso natural. Por esta razón, es importante dejar claro que el eje central de la acción de enfermería radica en el cuidado ofrecido a las personas, admitiéndose a las mismas, como sujetos que poseen derechos humanos universales, cuya atención gira alrededor del cumplimiento de éstos.

En este orden de ideas, es preciso mencionar a Henderson (1961): quien describe en su modelo de cuidados cuatro (4) principios bioéticos, que norman la relación terapéutica de enfermería con las personas que constantemente cuidan, los cuales son: 1). La beneficencia, definida como la obligación de hacer el bien; 2). La no maleficencia, entendida como la obligación de evitar hacer el mal a toda costa; 3). La Justicia; vista como la satisfacción de las necesidades básicas de las personas, sin importar las condiciones o los condicionamientos impuestos por la sociedad, lo que se valora es que sea humano; y 4). La Autonomía; la cual consiste en respetar a las personas como sujetos libres, aceptando sus decisiones y sus condiciones humanas, producto de sus valores y convicciones personales.

Cabe destacar que, los principios bioéticos fueron establecidos legalmente, por el Congreso de los Estados Unidos, en el año 1974, tal como lo señala Gómez (2009a): "[...] con el objetivo de proteger y garantizar los derechos de las personas incluidas en estudios de investigación biomédica, ya que ocasionalmente en el afán experimentador se llegaba a abusos" (pág. 231). Sin embargo, éstos, ya eran utilizados en el campo de la salud, tal como se nombró anteriormente.

En este sentido, concibieron a la beneficencia como la obligación de hacer el bien, siendo establecida como uno de los principios clásicos de la atención en salud, donde el actuar ético no demanda solamente el respeto de la libertad del otro, sino que incluye el objetivo del bien, estableciéndose que no se puede hacer un bien haciendo a la vez un daño; como ejemplo Gómez 
(2009b): menciona que "[...] el experimentar en humanos por el bien de la humanidad; no se puede hacer sin contar con el consentimiento de los sujetos, y menos sometiéndolos a riesgos o causando daño" (pág. 232).

La no maleficencia, está basada en el respeto de la integridad del ser humano, la cual se hace cada vez más relevante ante los avances técnicocientíficos, siendo éste un aspecto de la ética tradicional de los profesionales de salud, tal como lo señala Gómez (2009c): “[...] el médico no está obligado a seguir la voluntad del paciente si la juzga contraria a su propia ética [...] la posición moral del médico se inspira también en conceptos filosóficos o religiosos que sus pacientes no comparten [...]" (pág. 232).

La autonomía, la define como la obligación de respetar los valores y opciones personales, en aquellas decisiones básicas que le atañen, este principio para Gómez (2009d), se basa en el:

Consentimiento libre e informado en el que se asume al paciente como una persona libre de decidir sobre su propio bien y que este no le puede ser impuesto en contra de su voluntad por medio de la fuerza o aprovechándose de su ignorancia (págs. 231-232).

Es decir que, el paciente es una persona libre y autónoma para decidir cualquier aspecto relacionado a su cuerpo, su salud y a su vida, y jamás pudiese ser sometido en contra de su voluntad a experimentos o investigaciones por medio de engaños. Por ello, el profesional de enfermería debe garantizar el cumplimiento de este principio.

Y el último principio es la justicia, considerada por Gómez (2009e): como un aspecto que se vincula a la atención ecuánime que permita lograr un bienestar en las personas cuidadas, evitando, además, cualquier tipo de discriminación relacionada con el acceso a los recursos sanitarios, destinados para tal fin como políticas sociales del Estado, de modo que la población se sienta atendida y satisfecha en sus necesidades de salud. 
Es importante precisar que, los principios bioéticos son inspirados por las ciencias médicas, establecidos con el fin de fundamentar su actuación en los procesos inherentes a la biomedicina. Sin embargo, éstos son totalmente aceptados y aplicados en el ejercicio de la enfermería, debido a que la concepción del cuidado abarca una serie de aspectos que concatenan a éstos principios y establecen como propósito fundamental; velar por la preservación de la vida humana, basándose en el bienestar físico, mental, espiritual y social de la persona, sin importar sus condiciones o condicionamientos de la sociedad, por lo tanto, el profesional de enfermería está obligado a cumplir con todos éstos principios, sin poder desistir de su aplicación.

Por ende, más que seguir la voluntad del paciente, le corresponde aceptar y comprender ética y moralmente las acciones y decisiones que éstas y sus familiares asuman, destacando y demostrando el verdadero valor humano que caracteriza el cuidado de enfermería.

Este hecho, es lo que hace partícipe a los enfermeros y las enfermeras, de la esencia única que se basa en brindar cuidados humanos, a través del cumplimiento normo-ético de sus actos, siempre enfocados en el respeto por la vida y la salud de las personas, considerando al sujeto como un ser autónomo, con capacidad para tomar las decisiones en materia de su propia salud y bienestar, proteger su dignidad, ayudar al buen morir y en la aceptación de la muerte como un proceso natural. Destacando un conocimiento integral en asuntos religiosos o de libertad de credo, que permita desenvoltura y confianza en la influencia curativa espiritual, admitido desde la religión o creencia de las personas y sus familiares.

Por lo antes descrito, se considera que la práctica de enfermería dirige acciones que ponen de manifiesto el cumplimiento de principios éticos y morales, que norman la relación terapéutica e interpersonal, tales como la información, la veracidad, la confidencialidad, la intimidad, el respeto por las creencias o religiones, el respeto por los ideales y las elecciones de modo y 
estilos de vida de las personas, desde la actuación científico-técnica.

Concibiéndose además que, la bioética, conjuga los aspectos humanos en pro de brindar una atención terapéutica, basada en la satisfacción de las necesidades interferidas, donde la aplicación de principios bioéticos, van en consonancia con la dignificación de la vida, cuya acción los concibe como: la tolerancia, la benevolencia, la prudencia, la ecuanimidad, la fidelidad, la sinceridad, la diligencia, la confiabilidad y la solidaridad hacia las personas cuidadas y sus familiares, debido a que éstos conforman la estructura mental y la conciencia de los profesionales de enfermería, en cuanto al deber ser.

Bajo estos términos, se establece que el profesional de enfermería comprende al cuidado, como una acción que brinda asistencia y ayuda terapéutica, acogido en los valores que resultan del humanismo, como un acto de amor por y hacia las personas, por encima del usufructo o lucro y en pro del ser humano.

\section{Conclusiones}

Se puede establecer que el cuidar al otro es un acto consciente que denota la práctica de la ética de la vida y para la vida, por ende, la bioética es un elemento esencial en la realización de la práctica de enfermería. En este sentido, se establece que el cuidado humano es un proceso reflexivo, intersubjetivo, de sensaciones y experiencias compartidas, entre el profesional de enfermería y las personas cuya valoración sea exprese a través de necesidades humanas.

A esto se suma que, el cuidado es un acto de asistencia y ayuda terapéutica basada en una relación de respeto, un acto de amor por y hacia los humanos, y por ende es imprescindible realizar la práctica asistencial con esmero y dedicación, conjugando el humanismo por encima del usufructo o lucro, la inviolabilidad de la vida, implícito en el fomento y preservación de la salud y en la ayuda a la recuperación de la salud de las personas en condición 


\section{Ensayo Original / Original Essay}

de enfermedad.

La esencia del cuidado humano no debe ser contraria a los aspectos éticos de la vida, todo lo que encierra la colectividad y los procesos medioambientales, ya que se desintegra y se aleja de los deberes de las enfermeras y los enfermeros y los derechos humanos de las personas, donde aplica los principios bioéticos, como parte fundamental de los valores humanos que se emplea al realizar cuidados, es decir, que éstos principios orientan la actuación ética y humana para preservar inviolablemente la vida, reconociendo que las personas están compuestas por elementos físico, mental, espiritual y social, por ende son distintas e importantes, a tal punto que el respeto a su dignidad debe estar inmerso desde la actuación honesta, la aplicación de procedimientos, destacando la ayuda y satisfacción de necesidades, sin divulgar o permitir que se divulgue la intimidad corporal o información confidencial de las personas.

Es esa forma humana de tratar al otro, con ética, respeto, amor, una relación verdaderamente humana, que satisfaga las necesidades humanas y restablezca la salud de las personas objeto de nuestros cuidados, y, por ende, la protección de la vida.

Todo lo anteriormente señalado, justifica que el ejercicio de la enfermería está enfocado en los principios del humanismo, haciendo énfasis en la no discriminación por cultura, raza, sexo, posición ideológica y admitiendo una práctica ajustada en los aspectos éticos y bioéticos, ya que al profesional de enfermería le corresponde practicar la ética por y para las personas, la vida, el ser humano, como parte de una práctica humanista.

En este sentido, se considera que, para realizar una práctica de enfermería ajustada a los preceptos establecidos en la ley, es necesario tener una disposición positiva para hacerlo, ahí entra en juego la vocación de servicio y los valores humanos, realizando una ayuda humana con amor, como un acto de consciencia, reflexión sobre lo realizado, que se reconozca a los 
otros como personas que tienen una necesidad de ayuda, garantizando la aplicación de cuidados humanizados, tal como está establecido en los principios o normas deontológicas que rigen la materia, ya que los principios bioéticos marcan la esencia que acompaña al cuidado humano.

En la práctica de enfermería es fundamental demostrar un comportamiento ético, con y hacia las personas, como expresión del respeto a la vida, la dignidad humana y los valores; por ende, los principios bioéticos, conforman la articulación de los elementos humanos, vocacionales, los valores y las actitudes, como parte fundamental de dicha práctica, ya que el cuidado es realizado desde la comprensión integral del sujeto, respetando sus condiciones de vida y aplicando una serie de valores, que ayudan a dar respuesta a las necesidades humanas interferidas, de tal modo que se satisfagan las mismas.

Es por lo que, a los enfermeros y a las enfermeras, les corresponde realizar cuidados de calidad, basados en un clima de respeto tanto a la vida de las personas, como a la dignidad humana de éstas, que además inspire confianza en las personas cuidadas y sus familiares, por ello consideran necesario que los mismos se realicen desde la concepción y comprensión de los principios que rigen el derecho a la vida y la protección a la salud.

Se concibe una actuación consciente, que permita reflexionar y demostrar una actitud que se evidencie en un trato humano, con vocación de servicio, haciendo énfasis en la práctica de valores positivos descritos en el código deontológico, por lo tanto, los principios éticos de la vida, son parte fundamental del quehacer enfermero, ya que permite la satisfacción plena de las necesidades interferidas de las personas, familias y comunidades, con el fin último de proteger la vida.

Finalmente, se deduce que los principios bioéticos, conforman una serie de elementos que no sólo obliga a los enfermeros y enfermeras a cumplir éticamente la acción, sino que moralmente éstos son concebidos como parte 
del quehacer enfermero, por lo tanto, la esencia de la relación terapéutica debe tributar en la comprensión de las personas, desde su concepción biológica, espiritual, social y psicológica, donde la valoración reside principalmente en el respeto a la vida y la dignidad de las personas, aspectos esenciales de los principios bioéticos.

\section{Referencias}

FCPERBV (2008a,b). Código Deontológico de los Profesionales de la Enfermería de la República Bolivariana de Venezuela. IV Asamblea Nacional Extraordinaria. Maracay, Venezuela: Federación de Colegios de Profesionales de la Enfermería.

Gómez, P. (2009a,b,c,d,e). Principios básicos de bioética. Revista Peruana de Ginecología y Obstetricia, 55(4), 230-233, e-ISSN: 2304-5132. Recuperado de:

http://www.spog.org.pe/web/revista/index.php/RPGO/article/view/297 Henderson, V. (1961). CIE: Principios Básicos de los Cuidados de Enfermería. Publicaciones Científicas No. 57. Washington, D.C., Estados Unidos: Organización Panamericana de la Salud; Organización Mundial de la Salud. Recuperado de:

http://biblioteca.hsjdbcn.org/V\%20Henderson.pdf

Jahr, F. (2013). Essays in Bioethics 1924-1948. ISBN: 978-3643903372. Bochum, Germany: Lit Verlag.

RAE (2020). Consciencia. 23. ${ }^{a}$ edición. Madrid, España: Real Academia Española. Recuperado de: https://dle.rae.es/consciencia

Schmidt, L. (2008a,b). Van Rensselaer Potter: un paradigma de vida. Revista de Bioética Latinoamericana, 1(1), 1-21, e-ISSN: 2244-7482. Recuperado de: http://www.saber.ula.ve/handle/123456789/23750 Terán, C., \& Rico, Y. (2018). Mirada Axiológica al Discurso Formativo del Profesional de Enfermería. Revista Scientific, 3(9), 61-79, e-ISSN: 


\section{Ensayo Original / Original Essay}

2542-2987. Recuperado de:

https://doi.org/10.29394/Scientific.issn.2542-2987.2018.3.9.3.61-79

Vargas, M., \& Cortes, G. (2010). Una reflexión sobre valores y principios bioéticos en la práctica de Enfermería. Revista Mexicana de Enfermería Cardiológica, 18(1-2), 43-45, e-ISSN: 1405-0315. Recuperado de:

https://www.medigraphic.com/cgibin/new/resumen.cgi?IDARTICULO $=25686$

Wilches, A. (2011). La propuesta bioética de Van Rensselaer Potter, cuatro décadas después. Opción: Revista de Ciencias Humanas y Sociales, 27(66), 70-84, e-ISSN: 1012-1587. Recuperado de:

https://dialnet.unirioja.es/servlet/articulo?codigo $=3961004$ 


\section{Rubén Eliut Hernández Ortega}

e-mail: rubenho1977@gmail.com

Nacido en Güiria, estado Sucre, Venezuela, el 3 de

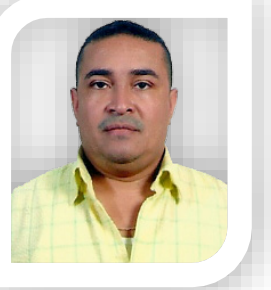
marzo del año 1977. Doctor en Ciencias de la Educación por la Universidad Latinoamericana y del Caribe (ULAC); Magister Scientiarum en Educación Superior por la Caribbean International University (CIU); Licenciado en Enfermería de la Universidad Nacional Experimental Rómulo Gallegos (UNERG); Director de los Programas Nacionales de Formación Avanzada en Enfermería de la Universidad de las Ciencias de la Salud (UCS, 2018); Coordinador Docente de la Dirección Nacional de Enfermería del Ministerio del Poder Popular para la Salud (2016-2018); Coordinador del PNF de Enfermería del Distrito Capital (2012-2016); Docente del PNF de Enfermería de la Universidad Politécnica Territorial de Los Altos Mirandinos "Cecilio Acosta" (UPTAMCA); Docente agregado con experiencia de 13 años; Enfermero con experiencia de 18 años; Miembro de la Línea de Investigación: Proceso de cuidado integral en la salud mental de las personas, familias y comunidades; Miembro del comité de diseño curricular de los postgrados en enfermería de la Universidad de las Ciencias de la Salud; Autor de artículos arbitrados en la Revista Scientific (e-ISSN: 2542-2987); Autor de la Tesis Doctoral: La Vocación del Profesional de Enfermería: Un Constructo Axiológico con Sentido Humanista (Mención Honorifica); Tutor de trabajos de investigación en pregrado, trabajo especial de grado y tesis doctoral.

El contenido de este manuscrito se difunde bajo una Licencia de Creative Commons ReconocimientoNoComercial-Compartirlgual 4.0 Internacional 